\title{
Optically pumped room temperature low threshold deep UV lasers grown on native AIN substrates
}

\author{
Akhil Raj Kumar Kalapala1†, Dong Liu ${ }^{2 \dagger}$, Sang June Cho ${ }^{2 \dagger,}$ \\ Jeongpil Park ${ }^{2 \dagger}$, Deyin Zhao ${ }^{1}$, John D. Albrecht ${ }^{4 *}$, Baxter Moody ${ }^{3 *}$, \\ Zhenqiang $\mathrm{Ma}^{2 *}$ and Weidong Zhou ${ }^{*}$
}

\begin{abstract}
We report here an optically pumped deep UV edge emitting laser with AIGaN multiple quantum wells (MQWs) active region grown on AIN substrate by low pressure organometallic vapor phase epitaxy (LP-OMVPE) in a high-temperature reactor. The 21 period $\mathrm{Al}_{0.53} \mathrm{Ga}_{0.47} \mathrm{~N}_{/} \mathrm{Al}_{0.7} \mathrm{Ga}_{0.3} \mathrm{~N}$ MQWs laser structure was optically pumped using $193 \mathrm{~nm}$ deep UV excimer laser source. A laser peak was achieved from the cleaved facets at $280.3 \mathrm{~nm}$ with linewidth of $0.08 \mathrm{~nm}$ at room temperature with threshold power density of $320 \mathrm{~kW} / \mathrm{cm}^{2}$. The emission is completely TE polarized and the side mode suppression ratio (SMSR) is measured to be around $14 \mathrm{~dB}$ at $450 \mathrm{~kW} / \mathrm{cm}^{2}$.
\end{abstract}

Keywords: optically pumped; AIGaN; UV edge emitting laser; AIN substrate

Kalapala A R K, Liu D, Cho S J, Park J P, Zhao D Y et al. Optically pumped room temperature low threshold deep UV lasers grown on native AIN substrates. Opto-Electron Adv 3, 190025 (2020).

\section{Introduction}

Since the first demonstration of laser diode (LD) on group III-nitride materials some 20 years ago, technology has significantly matured with the availability of high quality GaN and, recently, AlN substrates. This led to the demonstrations of high-performance laser diodes ranging from infrared to visible and down to the UV spectral regime claiming their applications in high-density data storage, spectroscopy, sterilization, chemical detection, communications, and manufacturing industry ${ }^{1,2}$. However, the development towards deep UV (<300 nm) lasers remains a great challenge mainly due to the lack of high quality single crystalline native AlN substrate. On the other hand, AlGaN devices grown on foreign substrates such as sapphire and silicon carbide suffer higher threading dislocation densities up to $10^{10} \mathrm{~cm}^{-2}$ and reduce the internal quantum efficiency. In addition, strain in AlGaN epitaxial layers due to lattice mismatch forms cracks when layers exceed critical thickness ${ }^{3-5}$. This results in an increase in non-radiative recombination centers, higher threshold and lower slope efficiency.

Optically pumped lasers based on AlGaN/AlN heterostructures at deep UV have recently been demonstrated by some groups using the MOCVD growth technique. Most of the reports are based on 3 to 10 periods of MQWs with threshold power densities ranging from 1200 $\mathrm{kW} / \mathrm{cm}^{2}$ down to $6 \mathrm{~kW} / \mathrm{cm}^{2}$. In 2004, a 3-pair $5 \mathrm{~nm} / 10$

\footnotetext{
${ }^{1}$ Department of Electrical and Computer Engineering, University of Texas at Arlington, Arlington, Texas 76019, United States; ${ }^{2}$ Department of Electrical and Computer Engineering, University of Wisconsin-Madison, Madison, Wisconsin 53706, United States; ${ }^{3} \mathrm{HexaTech}$, Inc., 991 Aviation Parkway, Suite 800, Morrisville, North Carolina 27560, United States; ${ }^{4}$ Department of Electrical and Computer Engineering, Michigan State University, East Lansing, Michigan 48824, United States.

${ }^{\dagger}$ These authors contributed equally to this work.

*Correspondence: W D Zhou, E-mail: wzhou@uta.edu; Z Q Ma, E-mail: mazq@engr.wisc.edu; B Moody, E-mail: baxter@adroitmaterials.com; J D Albrecht, E-mail: jalbrech@egr.msu.edu

Received: 3 July 2019; Accepted: 7 October 2019; Published: 20 April 2020
} 
nm $\mathrm{Al}_{0.66} \mathrm{Ga}_{0.34} \mathrm{~N} / \mathrm{Al}_{0.76} \mathrm{Ga}_{0.24} \mathrm{~N}$ MQWs LD structure was demonstrated by Takano et al at $241.5 \mathrm{~nm}$ with threshold of $1200 \mathrm{~kW} / \mathrm{cm}^{2}$ grown on $4 \mathrm{H}-\mathrm{SiC}$ substrate ${ }^{6}$. Wunderer in 2011 reported a 3-pair AlGaN MQWs laser structure with threshold of $126 \mathrm{~kW} / \mathrm{cm}^{2}$ at $267 \mathrm{~nm}^{7}$. In 2014, Guo et al reported a 10-pair $1.6 \mathrm{~nm} / 4 \mathrm{~nm} \quad \mathrm{Al}_{0.5} \mathrm{Ga}_{0.5} \mathrm{~N} /$ $\mathrm{Al}_{0.7} \mathrm{Ga}_{0.3} \mathrm{~N}$ MQW edge emitting laser at $265 \mathrm{~nm}$ with threshold of $150 \mathrm{~kW} / \mathrm{cm}^{28}$. The per QW threshold is calculated to be $15 \mathrm{~kW} / \mathrm{cm}^{2}$, but the full width half maximum (FWHM) is $1.8 \mathrm{~nm}$. Most recently in 2018, R. Kirste et al. reported a 3-period $2.5 \mathrm{~nm} \mathrm{Al} \mathrm{n}_{0.55} \mathrm{Ga}_{0.45} \mathrm{~N} / \mathrm{AlN}$ MQW laser at $267 \mathrm{~nm}$ with low threshold power density of 6 $\mathrm{kW} / \mathrm{cm}^{2}$ by reducing unintentional point defects in the active region through high supersaturation growth conditions for waveguide layer. However, the FWHM is still approximately $1 \mathrm{~nm}^{9}$. The threshold power density was decreased by increasing the $\mathrm{Al}$ composition in barriers thus increasing the barrier height to confine the carriers within the wells. These trends show a reduction in threshold power density by reducing the point defects, increasing the barrier height and optimizing the number of QWs and their thickness. However, the narrowest reported lasing linewidth is around $0.8 \mathrm{~nm}^{4,10}$. Similar to the threshold power density, the linewidth can also be further narrowed down with better growth quality of AlN epitaxial layers, thus increasing the laser characteristics.

In this work, we report a 21-period $2 \mathrm{~nm} \mathrm{Al}_{0.53} \mathrm{Ga}_{0.47} \mathrm{~N} / 6$ $\mathrm{nm} \mathrm{Al}_{0.7} \mathrm{Ga}_{0.3} \mathrm{~N}$ MQWs optically pumped laser on bulk AlN substrate by low pressure organometallic vapor phase epitaxy (LP-OMVPE) in a high-temperature reactor $^{5,11}$. The results show a dominant deep UV laser peak at $280.3 \mathrm{~nm}$, with lasing threshold of $320 \mathrm{~kW} / \mathrm{cm}^{2}$ and lasing FWHM of $0.08 \mathrm{~nm}$ at room temperature. Such a narrow spectral linewidth and low lasing threshold demonstrate high quality 21 QWs heterostructure growth.

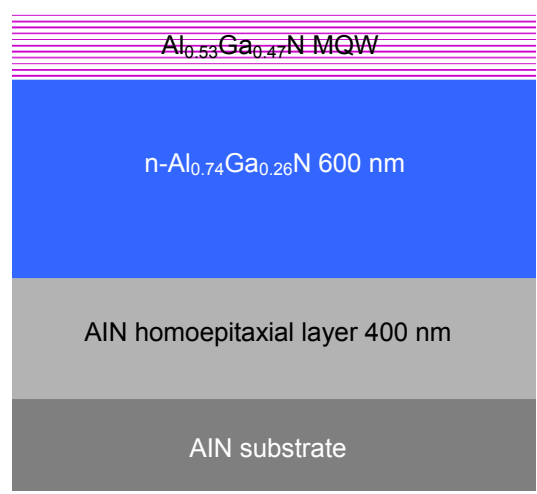

Fig. 1 | The deep UV laser heterostructure grown on AIN substrate with MQWs directly exposed to the air.

\section{Laser cavity design and fabrication}

Shown in Fig. 1 is the complete laser structure grown on an AlN substrate by LP-OMVPE in a high-temperature reactor. Following an initial $400 \mathrm{~nm}$ AlN homoepitaxial layer on the AlN substrate, a Si-doped $600 \mathrm{~nm}$ $\mathrm{n}-\mathrm{Al}_{0.74} \mathrm{Ga}_{0.26} \mathrm{~N}$ contact/cladding layer was grown prior to the 21-period $2 \mathrm{~nm} \mathrm{Al}_{0.53} \mathrm{Ga}_{0.47} \mathrm{~N} / 6 \mathrm{~nm} \mathrm{Al} \mathrm{Al}_{0.7} \mathrm{Ga}_{0.3} \mathrm{~N}$ MQWs active region. XRD was carried out to verify the material growth quality. Figure 2 shows the high-resolution XRD reciprocal space map. The measurement was carried out along (002) the direction with high-resolution triple axis scans. As shown in the RSM for a $21 \mathrm{AlGaN}$ QW grown on AlN substrate, high quality pseudomorphic growth was achieved with very close agreement with simulation for a completely pseudomorphic structure. Figure 3 shows the simulation of mode profile in the heterostructure with 3,15, and 21-period MQWs. This helps us to understand the confinement factor in the QWs and in turn the gain threshold value. We observed increments in the confinement factor as the number of QWs were increased. The heterostructure was designed to obtain a large modal confinement factor in the QW region. For the same heterostructure design, the optical mode confinement factor is stronger for 21 QWs with $18.51 \%$ for TE mode and $14.35 \%$ for TM mode respectively compared to 3 and 15-period QWs. Figure 5(b) shows the peak PL emission intensities of heterostructure designs with 3, 15 and 21 MQWs at different pumping power densities. It is observed that for a given pump power density, the peak PL emission increased linearly with the number of QWs. Therefore, 21-period MQW structure is used for the laser device fabrication.

The surface photoluminescence (PL) was measured to identify the QWs emission and gain region. A circular

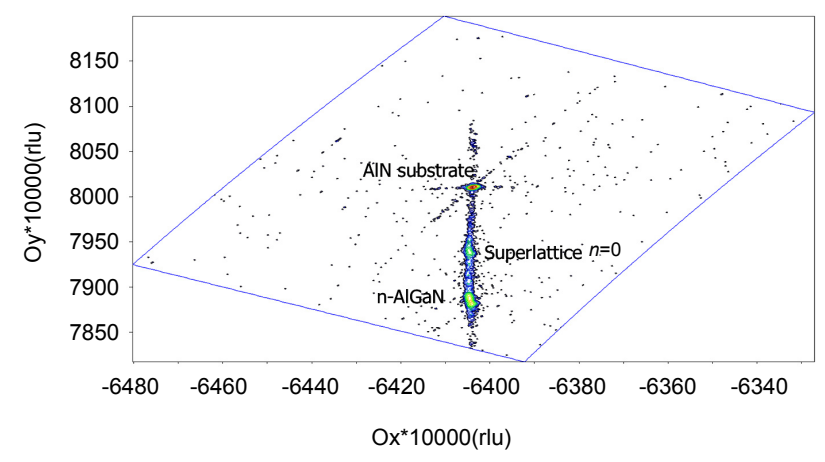

Fig. 2 | Pseudomorphic growth: High-resolution XRD reciprocal space map. 


\section{Opto-Electronic Advances https://doi.org/10.29026/oea.2020.190025}

$193 \mathrm{~nm}$ laser beam of $800 \mu \mathrm{m}$ diameter was incident on the sample and emission was collected at $45^{\circ}$ with respect to the incident beam. Figure 4 shows the surface photoluminescence experimental set up. We tested different sets of QW designs to compare the peak intensity and gain region. Table 1 shows the heterostructure information of each sample including the number of QWs, Al composition in QWs and barriers. The measured PL spectra from each sample are shown in Fig. 5(a). The emission intensity increased with the number of QWs as shown in Fig. 5(b). We considered sample S5 with emission peak centered at $273 \mathrm{~nm}$ to process our laser device as the emission from S5 sample is stronger than all the other samples at similar testing conditions. It is approximately 1.77 and 2.25 times stronger than S4 and S3 samples respectively. A subsequent chemical mechanical polishing (CMP) process was performed to thin down the substrate to around $70 \mu \mathrm{m}$.
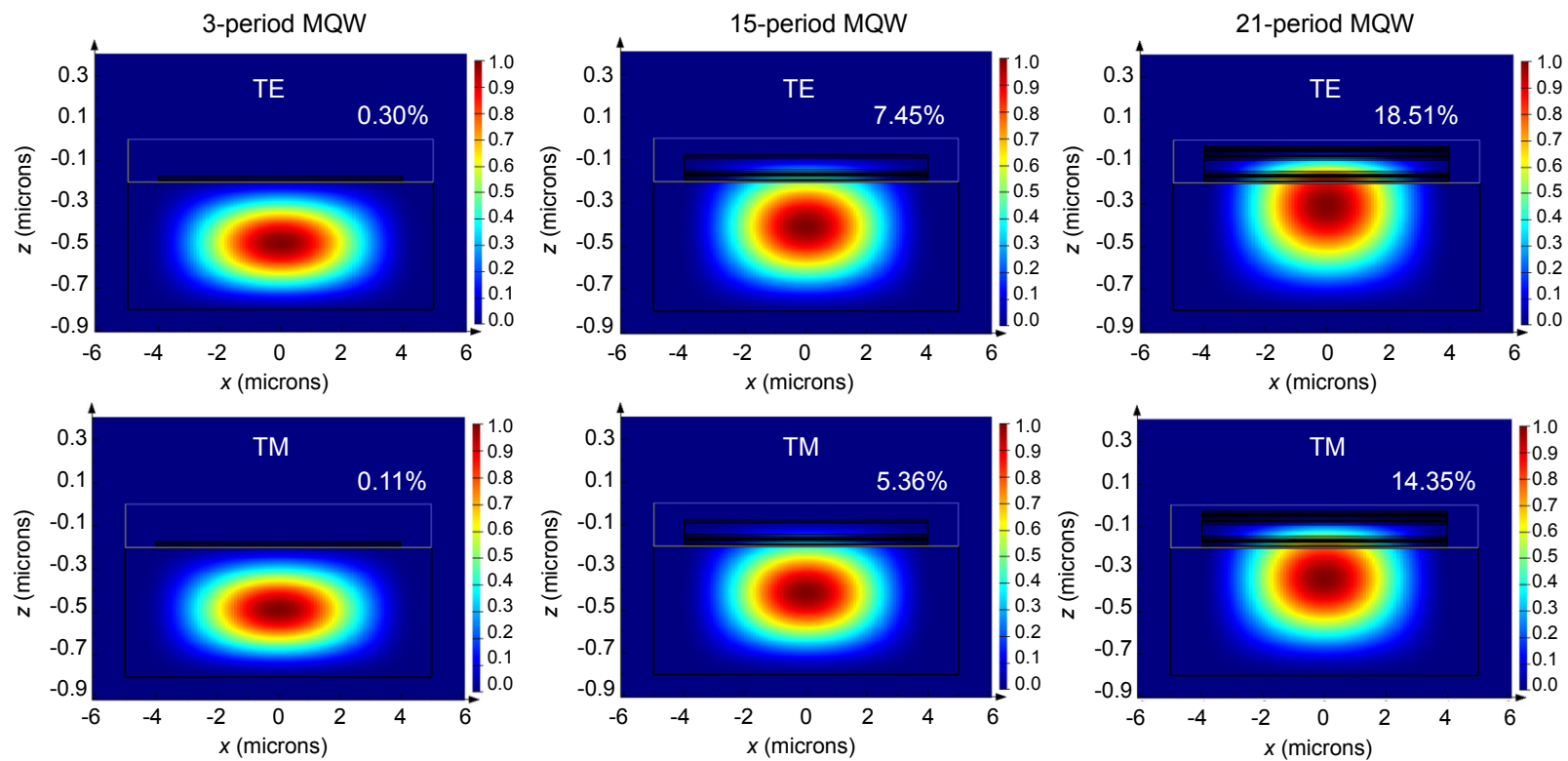

Fig. 3 | Simulations of TE and TM mode profile in the heterostructure with 3, 15 and 21-period MQWs.

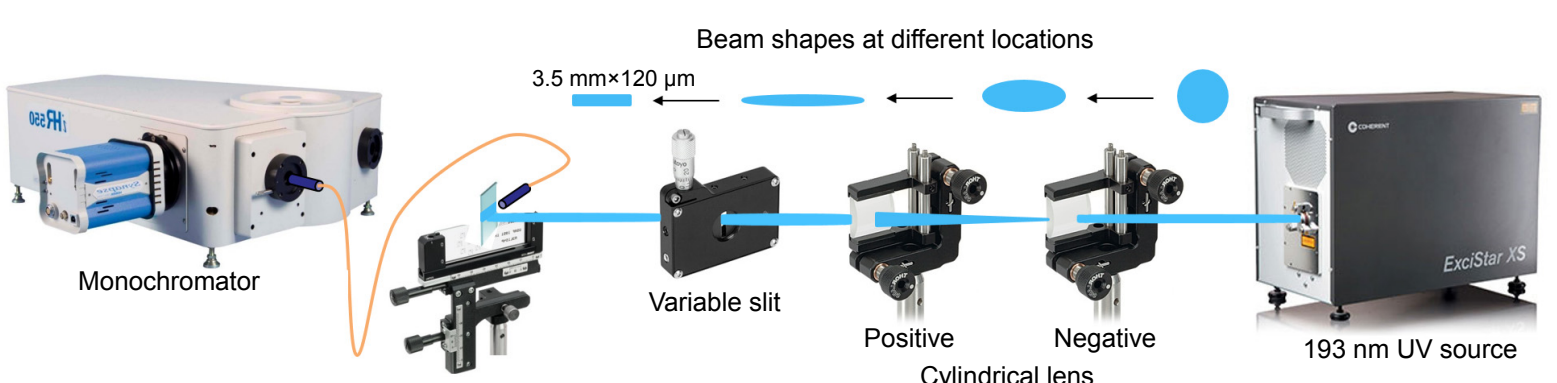

Fig. 4 | Schematic experimental setup to measure the edge emission from the cleaved facets.

Table 1 | Structure information for different sets of QW designs with different numbers of QWs and different Al compositions.

\begin{tabular}{c|c|c|c|c|c}
\hline Sample & \# of QWs & $\begin{array}{c}\text { QWs composition } \\
\mathrm{Al}_{x} \mathrm{Ga}_{1-x} \mathrm{~N}\end{array}$ & $\begin{array}{c}\text { QBs composition } \\
\mathrm{Al}_{x} \mathrm{Ga}_{1-x} \mathrm{~N}\end{array}$ & Peak wavelength (nm) & Sample name \\
\hline S1 & 3 & 0.75 & 1 & 234 & HT3761 \\
\hline S2 & 15 & 0.75 & 1 & 234 & HT5198 \\
\hline S3 & 21 & 0.72 & 1 & 234 & HT5294 \\
\hline S4 & 21 & 0.58 & 1 & 251 & HT5439 \\
\hline S5 & 21 & 0.53 & 0.7 & 273 & HT5630 \\
\hline
\end{tabular}



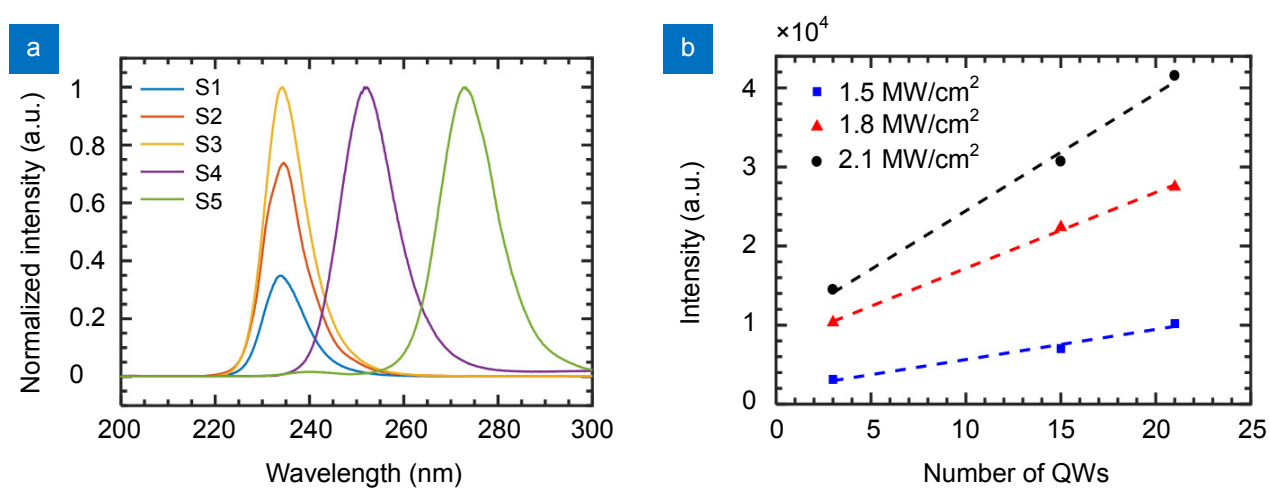

Fig. 5 | AIGaN QW growth on bulk AIN substrates. (a) Photoluminescence spectra for different sets of QW designs with different numbers of QWs and different Al compositions shown in Table 1; and (b) Photoluminescence intensity for different sets of QWs.

\section{Results and discussion}

The sample was then cleaved on one side to measure the net modal gain and loss using the variable-stripe length method. The stripe length is varied from $0.1 \mathrm{~mm}$ to $1 \mathrm{~mm}$ and emission from the facet is collected at various pump power densities. The onset of super-linearity is observed as the stripe length is increased from $0.5 \mathrm{~mm}$, as shown in Fig. 6. The net modal gain is calculated by fitting the peak

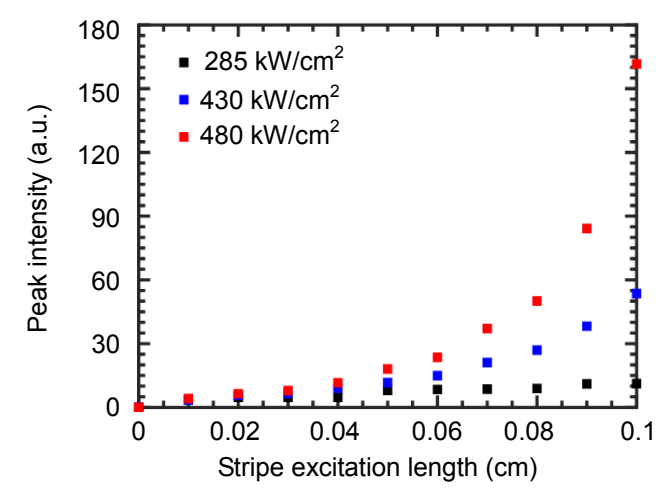

Fig. 6 | Peak emission intensities at different stripe excitation lengths for different pumping power densities. The onset of super-linearity after $0.5 \mathrm{~mm}$ excitation stripe length.

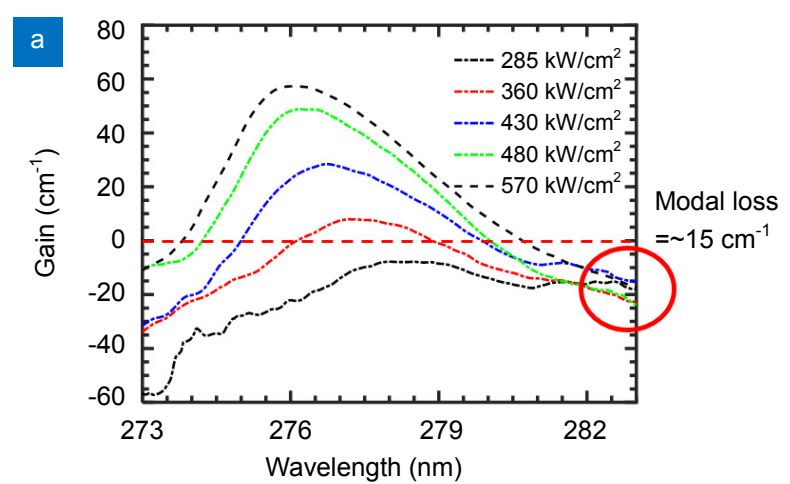

emission intensities vs stripe excitation length plot by using Matlab to the equation (1),

$$
I_{\mathrm{ASE}}(l, \lambda)=\frac{A}{G(\lambda)}\left[\mathrm{e}^{\mathrm{G}(\lambda) \cdot l}-1\right],
$$

where $I_{\mathrm{ASE}}$ is the amplified spontaneous emission intensity at different stripe lengths $l$ and wavelengths $\lambda, A$ is the constant related to the spontaneous emission intensity, and $G$ is the net optical gain as a function of wavelength ${ }^{8}$. Figure 7 (a) shows the gain spectra at different pump power densities. The net modal gain linearly increased with pump power up to $60 \mathrm{~cm}^{-1}$ and saturated from 500 $\mathrm{kW} / \mathrm{cm}^{2}$, as shown in Fig. 7(b).

Later, the other side was also cleaved to form two parallel facets, with a cavity length of $3.5 \mathrm{~mm}$. The QWs were directly exposed to the air on the top side with Si-doped cladding on the bottom. The laser structure was optically pumped using a coherent $193 \mathrm{~nm}$ ArF deep UV excimer laser source with a $7 \mathrm{~ns}$ pulse width and $200 \mathrm{~Hz}$ repetition rate. Cylindrical lenses were inserted in the pumping beam path to control the pump beam shape and beam uniformity. All tests were carried out at room temperature. The emission was collected from one of the cleaved edge facets into a fiber cable and coupled into an iHR550

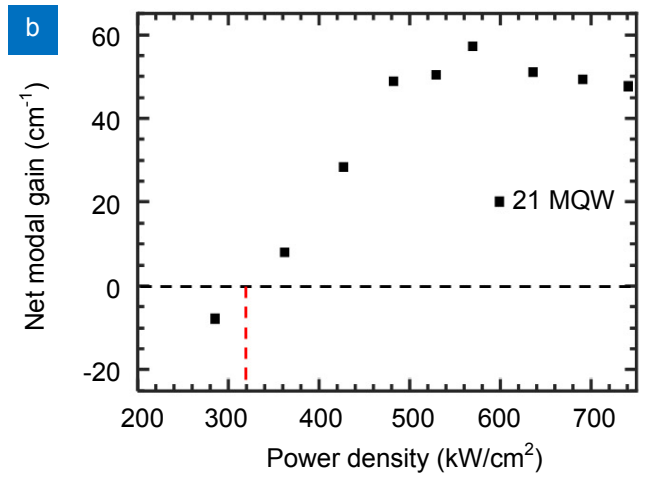

Fig. 7 | Optical gain measurement. (a) Measured gain spectra at different pump power densities, and (b) peak net modal gain at different pump power densities. 


\section{Opto-Electronic Advances https://doi.org/10.29026/oea.2020.190025}

monochrometer of 2400 groves $/ \mathrm{mm}$ grating with a spectral resolution of $0.01 \mathrm{~nm}$. Figure 8 (a) shows the measured spectral outputs at various pumping power densities with a pump beam width of $120 \mu \mathrm{m}$ over the entire laser cavity length of $3.5 \mathrm{~mm}$. Although the gain guided region is significantly broad, a dominant single mode lasing emission peak at $280 \mathrm{~nm}$ appeared above the threshold power density of $320 \mathrm{~kW} / \mathrm{cm}^{2}$. Figure $8(\mathrm{~b})$ shows the light-light (L-L) characteristics on primary $y$-axis and FWHM variation with pumping power density in secondary $y$-axis. The linewidth was observed to be 0.08 $\mathrm{nm}$, much narrower than any other DUV lasers reported so far (typ. $0.8 \mathrm{~nm})^{4,10}$. An elliptical shaped far-field pattern from the MQWs region was observed on the UV sensor card above the threshold at pumping power density of $350 \mathrm{~kW} / \mathrm{cm}^{2}$. The increase in the number of QWs has raised the threshold power density, but the threshold power density per QW is $15.2 \mathrm{~kW} / \mathrm{cm}^{2}$ which is still low for AlGaN MQWs structure with AlGaN barriers. These results with the low per QW threshold power density and
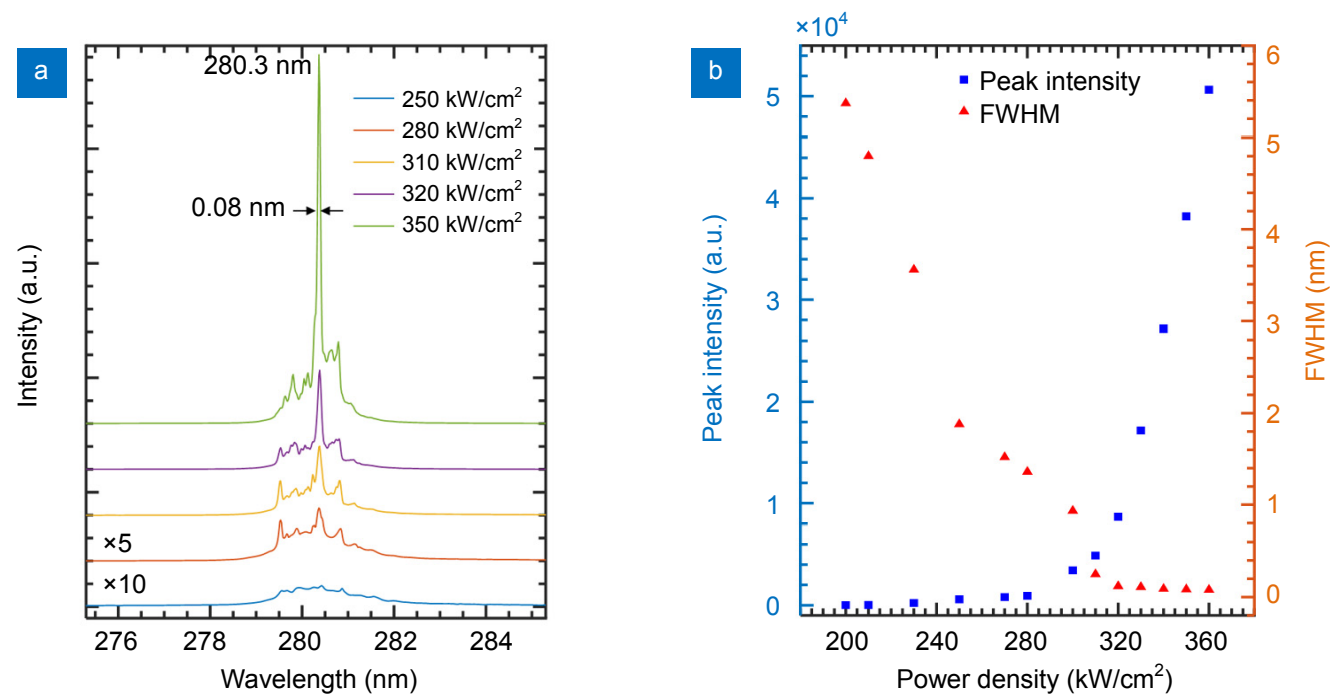

Fig. 8 Optically pumped laser emission measurement. (a) Edge emission spectra measured from the cleaved facet at different pump power levels. (b) Measured L-L curve and FWHM at different pumping power densities.
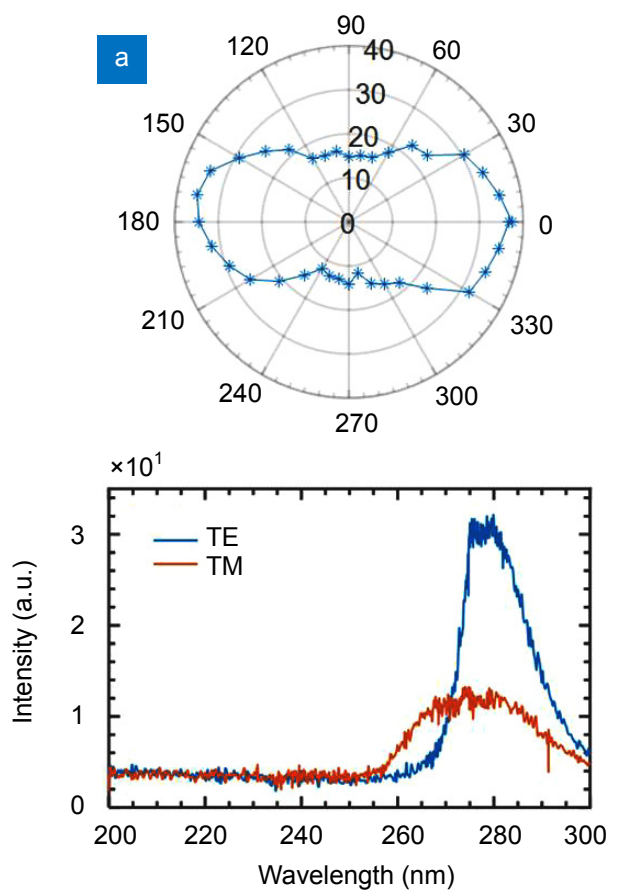
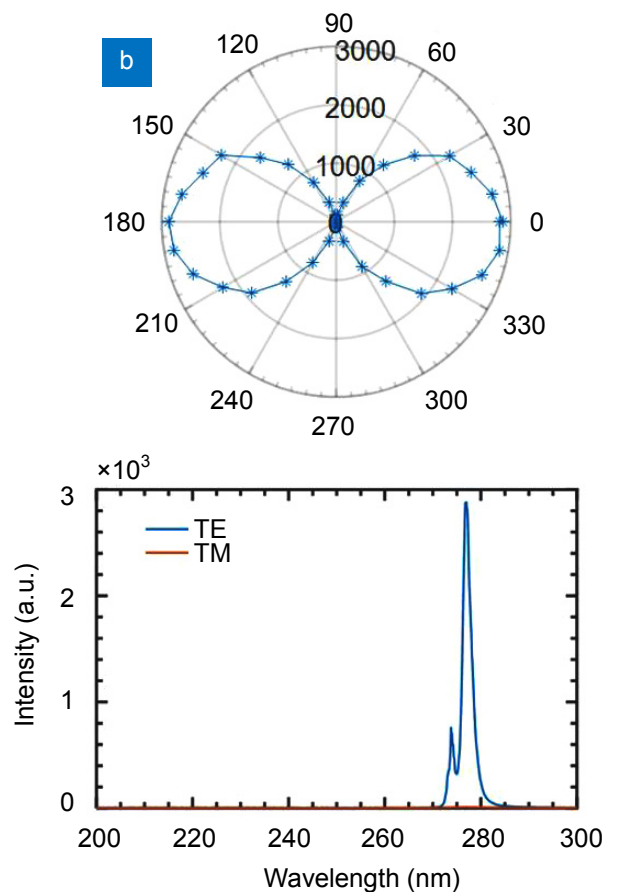

Fig. 9 | Polarization measurement (a) below threshold at $230 \mathrm{~kW} / \mathrm{cm}^{2}$ and (b) above threshold at $350 \mathrm{~kW} / \mathrm{cm}^{2}$. 


\section{Opto-Electronic Advances https://doi.org/10.29026/oea.2020.190025}

narrow linewidth suggest excellent QW heterostructure cavity and low optical loss in the processed laser structure.

Polarization properties were also measured, as shown in Fig. 9. Below threshold, we observed TE dominated emission with a TE/TM ratio of 2 . On the other hand, above threshold, the emission is $100 \% \mathrm{TE}$ polarized without any TM modes. These results are also in agreement with earlier reported work indicating dominant TE mode above $240 \mathrm{~nm}^{12}$.

\section{Conclusion}

We successfully demonstrated an optically pumped 280 $\mathrm{nm}$ deep UV edge emitting laser under pulsed operation at room temperature with a threshold of $320 \mathrm{~kW} / \mathrm{cm}^{2}$ and lasing linewidth of $0.08 \mathrm{~nm}$. The threshold per QW is only $15 \mathrm{~kW} / \mathrm{cm}^{2}$, suggesting high-quality crystal growth and processed laser cavity.

\section{References}

1. Nakamura S, Pearton S, Fasol G. The Blue Laser Diode: The Complete Story 2nd ed (Springer, Berlin Heidelberg, 2000).

2. Kinoshita $T$, Obata $T$, Nagashima $T$, Yanagi $H$, Moody $B$ et al. Performance and reliability of deep-ultraviolet light-emitting diodes fabricated on AIN substrates prepared by hydride vapor phase epitaxy. Appl Phys Express 6, 092103 (2013).

3. Yoshida H, Yamashita Y, Kuwabara M, Kan H. A 342-nm ultraviolet alGaN multiple-quantum-well laser diode. Nat Photonics 2, 551 (2008).

4. Xie J Q, Mita S, Bryan Z, Guo W, Hussey L et al. Lasing and longitudinal cavity modes in photo-pumped deep ultraviolet alGaN heterostructures. Appl Phys Lett 102, 171102 (2013).

5. Adivarahan V, Sun W H, Chitnis A, Shatalov M, Wu S et al. 250 nm AIGaN light-emitting diodes. Appl Phys Lett 85, 2175-2177 (2004).
6. Takano T, Narita Y, Horiuchi A, Kawanishi H. Room-temperature deep-ultraviolet lasing at $241.5 \mathrm{~nm}$ of $\mathrm{AlGaN}$ multiple-quantum-well laser. Appl Phys Lett 84, 3567-3569 (2004).

7. Wunderer T, Chua C L, Yang Z H, Northrup J E, Johnson N M et al. Pseudomorphically grown ultraviolet $C$ photopumped lasers on bulk AIN substrates. Appl Phys Express 4, 092101 (2011).

8. Guo W, Bryan Z, Xie J Q, Kirste R, Mita $S$ et al. Stimulated emission and optical gain in AIGaN heterostructures grown on bulk AIN substrates. J Appl Phys 115, 103108 (2014).

9. Kirste R, Guo Q, Dycus J H, Franke A, Mita $S$ et al. $6 \mathrm{~kW} / \mathrm{cm}^{2}$ UVC laser threshold in optically pumped lasers achieved by controlling point defect formation. Appl Phys Express 11, 082101 (2018).

10. Bryan Z, Bryan I, Kirste R, Collazo R, Sitar Z. Status and challenges in deep UV semiconductor lasers. In Proceedings of 2015 IEEE Summer Topicals Meeting Series (SUM) 123-124 (IEEE, 2015); http://doi.org/10.1109/PHOSST.2015.7248225.

11. Kalapala A R, Liu D, Cho S J, Park J, Zhao D Y et al. Optically pumped room temperature low threshold deep UV lasers grown on native AIN substrates. Proc SPIE 10918, 109180 (2019).

12. Northrup J E, Chua $C$ L, Yang Z, Wunderer T, Kneissl M et al. Effect of strain and barrier composition on the polarization of light emission from AIGaN/AIN quantum wells. Appl Phys Lett 100, 021101 (2012).

\section{Acknowledgements}

The work was supported by Defense Advanced Research Projects Agency (DARPA) under grant \# HR0011-15-2-0002. The program managers are Dr. Daniel Green and Dr. Young-Kai Chen.

\section{Author contributions}

Akhil Raj Kumar Kalapala, Dong Liu, Sang June Cho and Jeongpil Park contributed equally to this work.

All authors commented on the manuscript.

\section{Competing interests}

The authors declare no competing financial interests. 